\title{
Computational Challenges for Beam-Beam Simulation for RHIC
}

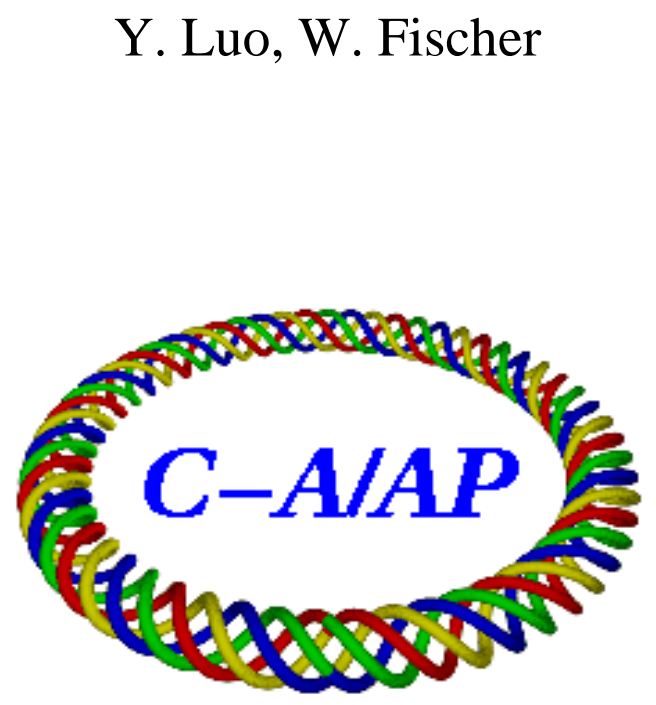

\section{Collider-Accelerator Department Brookhaven National Laboratory Upton, NY 11973}

Notice: This document has been authorized by employees of Brookhaven Science Associates, LLC under Contract No. DE-AC02-98CH10886 with the U.S. Department of Energy. The United States Government retains a nonexclusive, paid-up, irrevocable, world-wide license to publish or reproduce the published form of this 


\title{
COMPUTATIONAL CHALLENGES FOR BEAM-BEAM SIMULATION FOR RHIC*
}

\author{
Y. Luo, W. Fischer, Brookhaven National Laboratory, Upton, NY USA
}

\begin{abstract}
In this article we will review the computational challenges in the beam-beam simulation for the polarized proton run of the Relativistic Heavy Ion Collider (RHIC). The difficulties in our multi-particle and million turn tracking to calculate the proton beam lifetime and proton beam emittance growth due to head-on beam-beam interaction and head-on beam-beam compensation are presented and discussed. Solutions to obtain meaningful physics results from these trackings are proposed and tested. In the end we will present the progress in the benchmarking of the RHIC operational proton beam lifetime.
\end{abstract}

\section{INTRODUCTION}

The Relativistic Heavy Ion Collider (RHIC) accelerates and collides ions and polarized protons. For the experiments the figure of merit in the polarized proton run is $L P_{B}^{2} P_{Y}^{2}$, where $L$ is the luminosity and $P_{B, Y}$ are the polarizations of the Blue and Yellow beams respectively. Since its first polarized proton run at $100 \mathrm{GeV}$ in 2003 , the polarized proton luminosity has increased by an order of magnitude. And the proton polarization reached $55 \%$ and $34 \%$ at $100 \mathrm{GeV}$ and $250 \mathrm{GeV}$.

The main limits to the luminosity improvement in the RHIC polarized proton run are the beam-beam interaction effect, the nonlinear effect from the lattice, and the parameter modulations. To further increase the proton luminosity [1], we would like to reduce the $\beta^{*}$ at the interaction points from current $0.7 \mathrm{~m}$ to $0.5 \mathrm{~m}$, and to increase the bunch intensity from current $1.5 \times 10^{11}$ to $2.0 \times 10^{11}$ and perhaps beyond. An upgrade of the polarized proton source has been started to increase the proton current by an order of magnitude and the polarization by about $5 \%$ to $85-90 \%$.

For the polarized proton runs, the working point is chosen to provide good beam lifetime and maintain the proton polarization. The current working point is constrained between $2 / 3$ and $7 / 10$. When the proton bunch intensity is above $2 \times 10^{11}$, there will not be enough tune space between $2 / 3$ and $7 / 10$ to hold the beam-beam tune spread. One solution is to adopt head-on beam-beam compensation [2]. The idea is to introduce a low energy electron beam to collide with the proton beam to compensate the proton-proton beam-beam effects. Our preliminary simulation study shows that head-on beam-beam compensation with the e-lenses can significantly reduce the large beambeam tune spread. However, considering that the e-lenses

${ }^{*}$ This work was supported by Brookhaven Science Associates, LLC under Contract No. DE-AC02-98CH10886 with the U.S. Department of Energy. are strong nonlinear elements, their effects on the proton beam dynamics and lifetime have been carefully studied.

Since beam-beam effect has played a more and more important role in the polarized proton run in RHIC, numeric simulation studies are needed to understand the current RHIC operations and to predict the effect of head-on beam-beam compensation. In the article we will review the computational challenges in our beam-beam simulation. With limited computing resource and computing time, some approaches and new algorithm to reduce the statistic fluctuations in the calculated beam lifetime and emittance are presented and tested. Progress in the benchmarking of RHIC operational proton beam lifetime is also reported.

\section{CHALLENGES IN SIMULATION}

To reproduce the observations in the real operations, a robust simulation code and a realistic lattice model are needed. For RHIC, the lattice model should include the correct linear optics, all non-linear magnetic field errors, and all known parameter modulations.

To save the computing time, we adopt a weak-strong beam-beam model although the two proton beams have similar populations. Considering $\beta^{*}$ is comparable to the RMS bunch length at IP6 and IP8 in the polarized proton run, we adopt the 6-D weak-strong synchro-beam map a la Hirata to calculate the beam-beam kicks.

Our simulation code is SimTrack [3], which is a C++ library for the optics calculation and particle trackings in the high energy accelerators. The particle motion in the magnetic elements is tracked with the 4th order symplectic integration. To save computing time, multipoles are treated as thin lenses. Particles are tracked element by element.

Dynamic aperture has been frequently used to judge the stability of lattice and the effect of beam-beam interaction in RHIC [4]. Comparing to multi-particle tracking of a 6-D Gaussian distribution, it only needs a small amount of computing time. The shortcoming of dynamic aperture is that it does not give information of emittance evolution. And there is not a clear calibration between dynamic aperture and beam lifetime. Online measurement of dynamic aperture with beam is also time-consuming.

Actually in the operations of a collider, the beam intensity, the transverse and longitudinal beam sizes, and the luminosity are all directly measured. And in a longterm multi-particle tracking of a 6-D Gaussian distribution bunch, they are well defined and can be calculated too. Therefore, these parameters are suitable for the purpose of benchmarking simulation codes and comparing operation observations and simulation results. 
The challenge in the lifetime and emittance calculation is to obtain meaningful physics results with limited computing resources and computing time. However, to reduce the statistic errors in the calculations of beam lifetime and emittance, a large number of macro-particles is needed. And to determine the beam lifetime and the emittance growth, a large tracking turn is required.

\section{An example}

We first give an example to illustrate the computational challenges in the beam-beam simulation of lifetime and emittance calculations in RHIC. In this example, the bunch intensity is $2.5 \times 10^{11}$. The $\beta^{*}$ is $0.5 \mathrm{~m}$. The beam energy is $250 \mathrm{GeV}$. The beams collide at IP6 and IP8. We track 4800 macro-particles whose initial coordinates are sampled from a 6-D Gaussian distribution up to $2 \times 10^{6}$ turns. $2 \times 10^{6}$ turns is about 24 seconds of RHIC time. We record the number of lost particles and calculate the emittance at every $10^{4}$ turns. It turns out that there is only 1 macro-particle lost after $2 \times 10^{6}$ turns. There is no clear trend in the emittance change. And the fluctuation in the calculated emittance is about $2 \%$ of the averaged one.

Actually the lost particles in the tracking are normally those with large transverse amplitudes and large momentum deviations. For a Gaussian distribution with a limited number of macro-particles, there are only a few of macroparticles in the bunch tail. Therefore, to overcome the statistical error in the calculated particle loss rate and to better represent the particles in the tail of a 6-D Gaussian bunch, we need a large number of macro-particles. A large number of macro-particles in the tracking will significantly increase the computing time.

\section{Hollow Gaussian Distribution}

One approach is to track particles initially with a hollow Gaussian distribution [5,6]. This approach is suitable to calculate the particle loss rate. However it will not give information of emittance evolution. Since this approach is based on the assumption that the particles in the bunch core are not lost in the tracking turns, the boundary between the stable core and the unstable bunch tail needs to be carefully determined.

Normally we first calculate the dynamic aperture and then set the boundary well below it. After tracking, we also need to check if there are particles lost on the edge of boundary. If the boundary is chosen too low, there will be few particles lost in the tracking and the statistic error will be large. However if the boundary is chosen too high, there will be lost particles below the boundary are not counted. In this case the particle loss rate will be under estimated.

As a comparison, with the same simulation parameters in the above example, we track 4800 macro-particles whose initial amplitudes are larger than $3.0 \sigma$. Their coordinates can be generated from a normal Gaussian generator or a Gaussian tail generator. After $2 \times 10^{6}$ turn tracking, there are 16 macro-particles lost. In this example, the 4800 macro-particles actually represent 66269 particles of a 6-D solid Gaussian distribution. Although the computing time is the same as the Gaussian distribution, the statistic error in the particle loss rate is reduced. Figure 1 shows the transverse amplitudes of all and lost macro-particles with hollow Gaussian tracking.

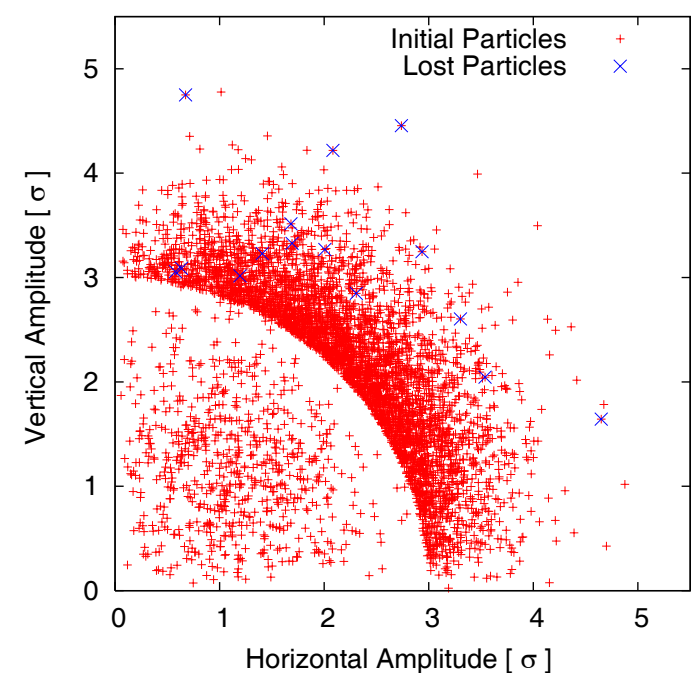

Figure 1: Initial transverse amplitudes of all and lost macro-particles with initial hollow Gaussian distribution.

\section{Weighted Gaussian Distribution}

Another approach is to track macro-particles initially with a 6-D weighted Gaussian distribution [7, 8]. In this approach, we designate a larger weight for the macroparticles in the bunch core while give a smaller weight for the macro-particles in the tail. With the same number of macro-particles, there will be more macro-particles in the bunch tail and a less number of macro-particles in the bunch center. This approach also gives the emittance besides the particle loss rate although its resolution is better than that with the plain Gaussian distribution.

With the same beam and lattice condition, with the weighted Gaussian distribution, there are 20 particles lost after $2 \times 10^{6}$ turns. The 4800 macro-particles of a weighted Gaussian actually represented 70108 particles of a 6-D Gaussian distribution. Table 1 lists the number of lost macro-particles and the beam decay. To get the beam decay per hour, we need to multiply the relative beam loss in $2 \times 10^{6}$ turns by 140 . From Table 1 , the beam decays from the hollow and weighted Gaussian distortions are very close. Figure 2 shows the transverse amplitudes of all and lost macro-particles with weighted Gaussian distribution tracking.

\section{Emittance Calculation}

The challenge in the emittance calculation is that the real emittance growth of the proton beam in $2 \times 10^{6}$ turns is very small and therefore difficulty to detect. In our simulation, 
Table 1: Particle Losses with Different Initial Distributions

\begin{tabular}{lccc}
\hline \hline Case & $N_{\text {represent }}$ & $N_{\text {lost }}$ & beam decay \\
\hline Plain Gaussian & 4800 & 1 & $2.9 \% / \mathrm{hr}$ \\
Hollow Gaussian & 66269 & 16 & $3.4 \% / \mathrm{hr}$ \\
Weighted Gaussian & 70108 & 20 & $4.0 \% / \mathrm{hr}$ \\
\hline \hline
\end{tabular}

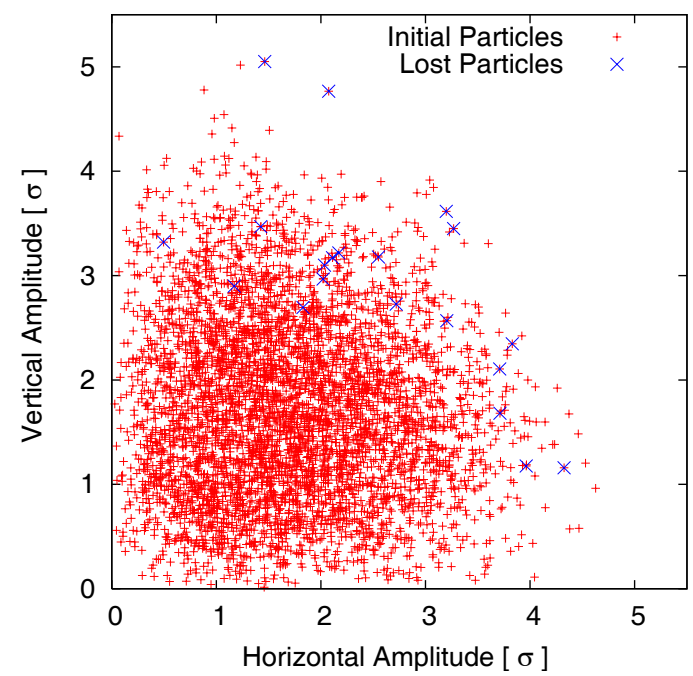

Figure 2: Initial transverse amplitudes of all and lost macro-particles with initial weighted Gaussian distribution.

we found that the calculated emittance is affected by the particle loss and the large amplitude macro-particles. The straight-forward way to reduce the fluctuation in the calculated emittance is to increase the total number of particles in tracking. However, limited by the computing time, this is not easily possible. And the statistic error in the emittance calculation will not be significantly reduced even if we increase the total number of macro-particles by one or two orders.

A new algorithm to calculated the emittance was implemented in LIFETRAC code. It calculate the emittance with all the coordinates of all macro-particles in all turns in each step of $10^{4}$ turns. By doing that, the statistic error in the emittance calculation will be reduced by a factor of 100. Figure 3 shows the calculate emittance without or with this algorithm in the above examples. The number of macro-particles is still 4800. From Figure 3, this algorithm greatly reduces the fluctuation in the calculated emittance for both Gaussian and weighted Gaussian distribution trackings. With the new algorithm, the fluctuation in the calculated emittance is about $0.03 \%$ of the averaged one.

\section{BENCHMARKING RHIC LIFETIME}

In the simulation studies of the effect of head-on beambeam compensation in RHIC, we systematically calculated and compared the particle loss rate without and with head-on beam compensation. The simulation shows that

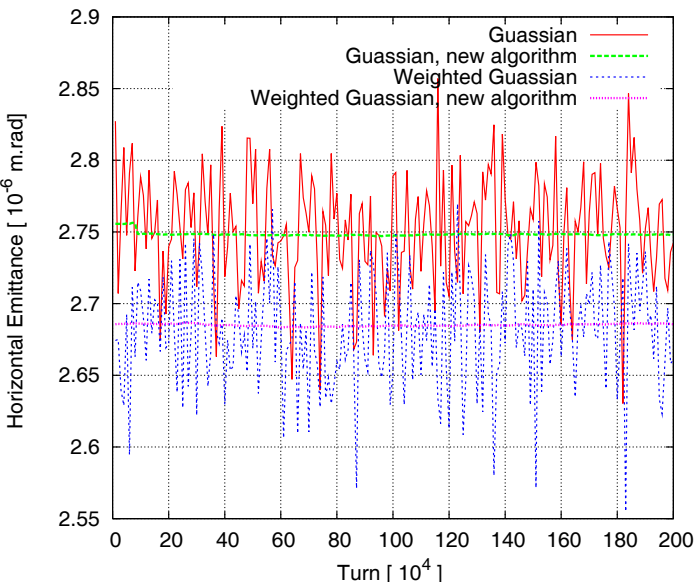

Figure 3: Calculated horizontal emittance without and with new algorithm for Gaussian and weighted Gaussian distributions.

half head-on beam-beam compensation improves the proton lifetime when the bunch intensity is above $2.0 \times 10^{11}$. In these studies we used hollow Gaussian approach to produce more macro-particle losses. The calculated beam decay with half beam-beam compensation for bunch intensity $2.5 \times 10^{11}$ is about less than $1 \%$.

To benchmark our simulation code and algorithms, we calculated and compared the proton lifetime and emittance with the previous polarized proton operations. Due to the small beam-beam parameter, the particle loss from beambeam interaction is very small and very hard to detect in the simulation even we push up very high the boundary between the stable core and unstable tail in the hollow Gaussian approach. In the real operations, the observed beam loss at store was about a few percent per hour.

To fill the gap in the proton lifetime between the simulation results and the real observations, we included the multipole field errors in the arc dipoles and quadrupoles as well as the interaction region multipole errors. The known tune modulations are also included in the lattice. Currently we are updating the tracking model according to the online optics measurement and modeling, and investigating the effects from other diffusion processes, such as the beam-gas scattering, intra-beam scattering and other noises.

\section{REFERENCES}

[1] W. Fischer, in Proceedings of IPAC'2010, Kyoto, Japan.

[2] Y. Luo, W. Fischer, BNL C-AD AP Note 286, 2007.

[3] Y. Luo, BNL C-AD AP Note 388, 2010.

[4] Y. Luo, et al., in Proceedings of PAC'2009, Vancouver, Canada.

[5] Y. Luo, et al., in Proceedings of EPAC'2008, Genoa, Italy.

[6] Y-J. Kim, et al., Phys. Rev. ST Accel. Beams 12, 031001 (2009).

[7] D. Shatilov, in Proceedings of PAC'2005, Knoxville, TN.

[8] A. Valishev, in Proceedings of PAC'2005, Knoxville, TN. 